PROCEEDINGS OF THE

AMERICAN MATHEMATICAL SOCIETY

Volume 134, Number 5, Pages 1369-1374

S 0002-9939(05)08300-0

Article electronically published on October 6, 2005

\title{
ON THE OPEN SET CONDITION FOR SELF-SIMILAR FRACTALS
}

\author{
CHRISTOPH BANDT, NGUYEN VIET HUNG, AND HUI RAO
}

(Communicated by David Preiss)

\begin{abstract}
For self-similar sets, the existence of a feasible open set is a natural separation condition which expresses geometric as well as measure-theoretic properties. We give a constructive approach by defining a central open set and characterizing those points which do not belong to feasible open sets.
\end{abstract}

\section{INTRODUCTION}

1.1. Self-similar sets. Let $f_{1}, \ldots, f_{m}$ be contracting similarity maps on $\mathbb{R}^{n}$, that is,

$$
\left|f_{i}(x)-f_{i}(y)\right|=r_{i} \cdot|x-y| \text { for all } x, y \in \mathbb{R}^{n},
$$

where the $r_{i} \in(0,1)$ are the contraction factors and $|\cdot|$ denotes the Euclidean norm. There is a unique compact set $A$ which satisfies the set equation

$$
A=f_{1}(A) \cup \ldots \cup f_{m}(A)
$$

and is called the self-similar set generated by the maps $f_{i}$ [3, 2]. The set $A$ consists of similar copies $A_{i}=f_{i}(A)$ of itself, where each $A_{i}$ consists of smaller copies $A_{i j}=f_{i}\left(f_{j}(A)\right)$, and so on. For any integer $n$, we can consider the set $S^{n}$ of words $\mathbf{i}=i_{1} \ldots i_{n}$ from the alphabet $S=\{1, \ldots, m\}$. Writing $f_{\mathbf{i}}=f_{i_{1}} \ldots f_{i_{n}}$ and $A_{\mathbf{i}}=f_{\mathbf{i}}(A)$, we can express $A$ as

$$
A=\bigcup\left\{A_{\mathbf{i}} \mid \mathbf{i} \in S^{n}\right\} .
$$

When $n$ tends to infinity, this induces a continuous map $\pi: S^{\infty} \rightarrow A$ from the set $S^{\infty}$ of sequences $i_{1} i_{2} i_{3} \ldots$ onto the self-similar set, the so-called address map.

The open set condition (OSC). The $f_{i}$ are said to satisfy the OSC if there exists a nonempty open set $V \subset \mathbb{R}^{n}$ such that

$$
\bigcup_{i=1}^{m} f_{i}(V) \subseteq V \quad \text { and } \quad f_{i}(V) \cap f_{j}(V)=\emptyset \text { for } i \neq j .
$$

We call $V$ a feasible open set of the $f_{i}$, or of $A$. The OSC controls the overlap of the $A_{i}$. It was introduced by P.A.P. Moran [5] in 1946 in order to show that the

Received by the editors October 26, 2004, and, in revised form, December 2, 2004.

2000 Mathematics Subject Classification. Primary 28A80; Secondary 28A75.

Key words and phrases. Self-similar, fractals, iterated function system.

The third author was supported by the German Research Foundation (DFG), and the second author was supported by the Vietnamese Government and the German Academic Exchange Service (DAAD). 


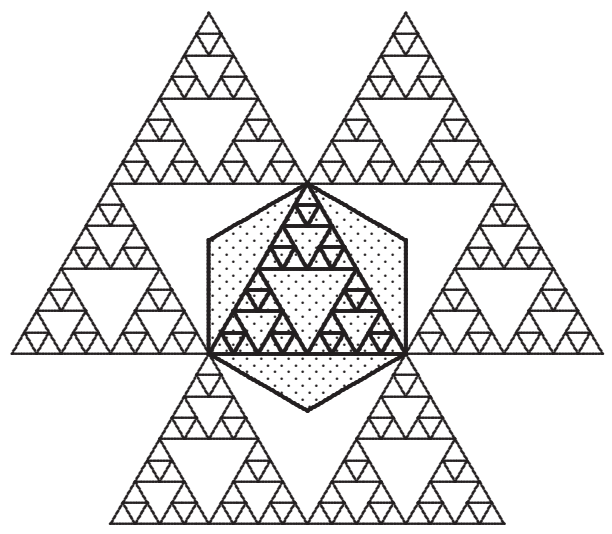

FiguRE 1. Some neighbor sets and central open set for the Sierpinski gasket.

canonical Hausdorff measure is positive on A. More recently, Schief [6] proved the converse: positive Hausdorff measure implies OSC. They also proved that OSC is equivalent to a combinatorial condition: there exists an integer $N$ such that at most $N$ incomparable pieces $A_{\mathbf{j}}$ of size $\geq \varepsilon$ can intersect the $\varepsilon$-neighborhood of a piece $A_{\mathbf{i}}$ of diameter $\varepsilon$.

The neighbor map condition. An algebraic equivalent for OSC was given by Bandt and Graf [1]. The inverse map $f_{\mathbf{i}}^{-1}$ is used to transform the small pieces $f_{\mathbf{i}}(A)$ and $f_{\mathbf{j}}(A)$ into $A$ and $h(A)=f_{\mathbf{i}}^{-1} f_{\mathbf{j}}(A)$, respectively. Here $h(A)$ is "the potential neighbor set" of $A$ if we imagine that the self-similar structure is extended outward from $A$. The second requirement of the OSC can be written as $f_{\mathbf{i}}(V) \cap f_{\mathbf{j}}(V)=\emptyset$ for $i_{1} \neq j_{1}$, and this is equivalent to $V \cap f_{\mathbf{i}}^{-1} f_{\mathbf{j}}(V)=\emptyset$. If such an open set $V$ exists, then the map $h=f_{\mathbf{i}}^{-1} f_{\mathbf{j}}$ cannot be near the identity map $i d$. Let $S^{*}=\bigcup_{n \geq 1} S^{n}$. The maps in

$$
\mathcal{N}=\left\{h=f_{\mathbf{i}}^{-1} f_{\mathbf{j}} \mid \mathbf{i}, \mathbf{j} \in S^{*}, i_{1} \neq j_{1}\right\}
$$

will be called neighbor maps. The algebraic formulation of OSC reads as follows.

There is a constant $\kappa>0$ such that $\|h-i d\|>\kappa$ for all neighbor maps $h$.

The norm of an affine mapping $g$ on $\mathbb{R}^{n}$ is $\|g\|=\sup _{|x| \leq 1}|g(x)|$, as usual. Geometrically, the condition says that compared to their size, two pieces $A_{\mathbf{i}}$ and $A_{\mathbf{j}}$ cannot be arbitrarily close to each other.

\section{The CEnTRAL OPEN SET}

So far, no algorithm is known to construct feasible open sets. Here we give a constructive approach. Let us say that $x \in \mathbb{R}^{n}$ is a forbidden point for $A$ if there is no feasible open set $V$ containing $x$.

All points of a "neighbor set" $h(A)=f_{\mathbf{i}}^{-1} f_{\mathbf{j}}(A)$ are forbidden points for $A$. This will follow from Proposition 3 and can easily be proved directly. Thus an open set $V$ cannot contain points of the set $H=\bigcup\{h(A) \mid h \in \mathcal{N}\}$. So let us define the central open set for $f_{1}, \ldots, f_{m}$ as

$$
V_{c}=\{x \mid d(x, A)<d(x, H)\},
$$



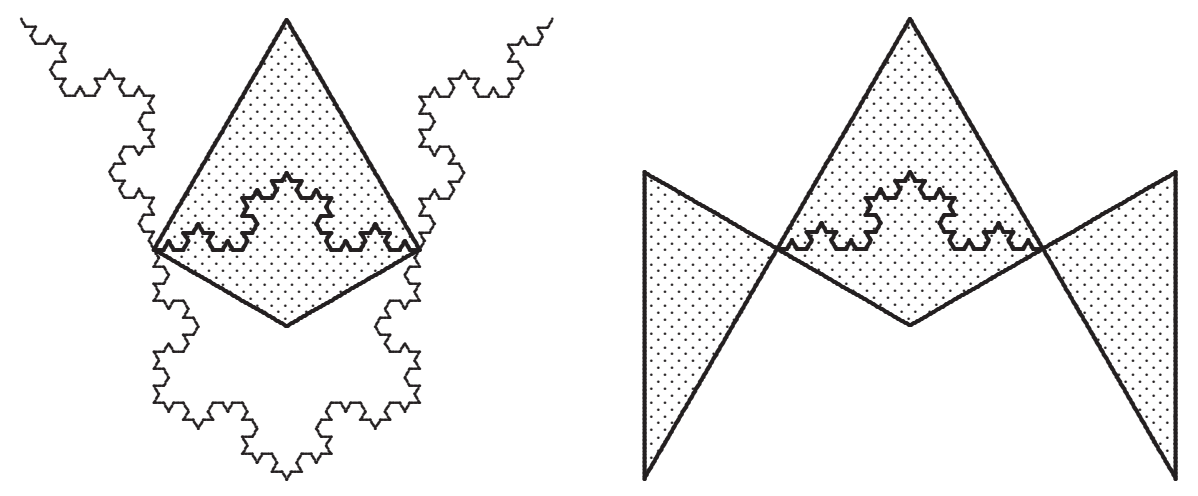

FiguRE 2. a) Neighbor sets and central open set for the Koch curve.

b) A feasible open set which contains the central one.

where $d(x, Y)=\inf \{|x-y| \mid y \in Y\}$ denotes the distance from a point to a set. Thus $d(x, H)=\inf \left\{d(x, h(A) \mid h \in \mathcal{N}\}\right.$. The definition of $V_{c}$ resembles the construction of a fundamental domain for a transformation group, where the neighbor maps play the part of the transformations.

Theorem 1. If OSC holds, the central open set $V_{c}$ is a feasible open set. If OSC does not hold, then $V_{c}$ is empty.

Proof. We show that $V_{c}$ fulfils condition (1) whenever it is nonempty. It follows that if OSC does not hold, $V_{c}$ must be empty. To verify $f_{i}\left(V_{c}\right) \subseteq V_{c}$, consider a point $x \in V_{c}$ and $y=f_{i}(x)$. Since $f_{i}$ is a similarity map with factor $r_{i}$,

$$
d(y, A) \leq d\left(y, f_{i}(A)\right)=r_{i} \cdot d(x, A)<r_{i} \cdot d(x, H)=d\left(y, f_{i}(H)\right) \leq d(y, H) .
$$

In the last step, we used $H \subseteq f_{i}(H)$ which follows from the fact that each $f_{\mathbf{i}}^{-1} f_{\mathbf{j}}(a)$ can be written as $f_{i}\left(f_{i}^{-1} f_{\mathbf{i}}^{-1} f_{\mathbf{j}}(a)\right)$.

Next, let $i \neq j$ and $x \in V_{c}$. Then $d(x, A)<d\left(x, f_{i}^{-1} f_{j}(A)\right)$ by definition of $V_{c}$. Applying $f_{i}$ on both sides of the inequality and dividing by $r_{i}$, we obtain

$$
d\left(f_{i}(x), f_{i}(A)\right)<d\left(f_{i}(x), f_{j}(A)\right) .
$$

The points of $f_{i}\left(V_{c}\right)$ are nearer to $A_{i}$ than to any other piece of $A$. A similar statement holds for the points of $f_{j}\left(V_{c}\right)$. This proves $f_{i}\left(V_{c}\right) \cap f_{j}\left(V_{c}\right)=\emptyset$.

If OSC holds, then by Schief [6] there is an open set $V$ with $A \cap V \neq \emptyset$. Since $V$ does not intersect $\bar{H}$ (cf. Proposition 3 ), each point in $V \cap A$ belongs to $V_{c}$, and so $V_{c}$ is nonempty.

Corollary 2. OSC holds if and only if $A$ is not contained in $\bar{H}$.

M. Moran [4 even claimed that OSC holds if and only if $H$ contains no dense subset of $A$, that is, $A \neq \overline{A \cap H}$. But since his proof contains a gap, his assertion remains open as long as we do not know more about $H$. So let us study the structure of forbidden points of $A$ in greater detail. 


\section{THE FIXED POINTS OF NEIGHBOR MAPS}

Hutchinson [3] characterized the self-similar set $A$ as the closure of the set of fixed points of the $f_{\mathbf{i}}, \mathbf{i} \in S^{*}$. It turns out that the set of forbidden points has a similar structure. Let $J$ denote the set of fixed points of neighbor maps,

$$
J=\left\{x \in \mathbb{R}^{n} \mid h(x)=x \text { for some } h \in \mathcal{N}\right\} .
$$

Proposition 3. All points of the closure $\bar{J}$ are forbidden points for $A$, and $H \subseteq \bar{J}$.

Proof. Assume $x \in \bar{J}$ belongs to an open set $V$. Then $V$ contains the fixed point $y$ of a neighbor map $f_{\mathbf{i}}^{-1} f_{\mathbf{j}}$. Thus $f_{\mathbf{i}}(V) \cap f_{\mathbf{j}}(V)$ contains $f_{\mathbf{i}}(y)$, so $V$ is not feasible.

To show that $H \subseteq \bar{J}$ we fix a point $b \in H$, say $b \in f_{\mathbf{i}}^{-1} f_{\mathbf{j}}(A)$. We assume that $|\mathbf{i}|<|\mathbf{j}|$ so that $h=f_{\mathbf{i}}^{-1} f_{\mathbf{j}}$ is contractive. Actually we may enlarge $\mathbf{j}$ as far as we want, for the only requirement is that $f_{\mathbf{i}}(b) \in f_{\mathbf{j}}(A)$.

Let $c$ be the fixed point of the map $h=f_{\mathbf{i}}^{-1} f_{\mathbf{j}}$. For $a=h^{-1}(b)$ we have

$$
|c-b|=|h(c)-h(a)|=r_{h} \cdot|c-a| .
$$

We fix $\mathbf{i}$ and extend $\mathbf{j}$ to obtain a sequence of $h_{n}$ such that $b \in h_{n}(A)$. In this procedure, one can show that $c$ is bounded, $r_{h}$ tends to 0 , and $a$ is bounded since it is in $A$. Therefore, the fixed points of $h_{n}$ tend to $b$.

Our problem here is whether there are forbidden points outside $\bar{J}$. For self-similar sets on the line, we will prove that there are no such points. When OSC holds, our statement is a bit more general. We start with a simple observation.

Lemma 4 (cf. 1], Prop. 1(i)). Given a forbidden point $x$ of $A$ and an $\varepsilon>0$, there is a neighbor map $h$ with $|h(x)-x|<\varepsilon$.

Proof. Let $B$ denote the open ball with center $x$ and radius $\varepsilon / 2$, and consider $V=\bigcup_{\mathbf{i} \in S^{*}} f_{\mathbf{i}}(B)$. Since $V$ is not a feasible open set, $f_{i}(V) \cap f_{j}(V) \neq \emptyset$ for some $i \neq j$. So there are $\mathbf{i}, \mathbf{j} \in S^{*}$ with $f_{i} f_{\mathbf{i}}(B) \cap f_{j} f_{\mathbf{j}}(B) \neq \emptyset$. The map $h=f_{i \mathbf{i}}^{-1} f_{j \mathbf{j}}$ fulfils the condition if $h$ is contractive; otherwise $h^{-1}$ fulfils the condition.

Theorem 5. Let the mappings $f_{i}(x)=u_{i} x+v_{i}$ on $\mathbb{C}^{n}$ with $u_{i} \in \mathbb{C}$ and $v_{i} \in \mathbb{C}^{n}$ satisfy OSC. Then any forbidden point belongs to $\bar{J}$.

Proof. Any neighbor map can be written as

$$
h(x)=r x+(1-r) c \text { or } h(x)=x+b \text { with } r \in \mathbb{C} \text { and } c, b \in \mathbb{C}^{n} .
$$

Let $x$ be a forbidden point. OSC means $\|h-i d\|>\kappa$ for all neighbor maps and some $\kappa>0$. Let $\varepsilon<\kappa / 2$ be given, and take $h$ from Lemma 4 , $h$ cannot be a translation since then $\|h-i d\|=|h(x)-x|$. Thus

$$
\varepsilon>|h(x)-x|=|1-r| \cdot|c-x| .
$$

By the definition of norm, there is a $y$ with $|y| \leq 1$ such that

$$
\kappa \leq|h(y)-y|=|1-r| \cdot|c-y| \cdot
$$

Subtracting the two inequalities and dividing by $|1-r|$, we obtain

$$
(\kappa-\varepsilon) /|1-r|<|c-y|-|c-x| \leq|x-y| \leq|x|+1 .
$$


Now we use the first inequality again:

$$
|c-x|<\frac{\varepsilon}{|1-r|}<\frac{\varepsilon}{\kappa-\varepsilon} \cdot(|x|+1)<\frac{2 \varepsilon}{\kappa} \cdot(|x|+1) .
$$

For $\varepsilon \rightarrow 0$ this shows that $x$ is in $\bar{J}$.

Theorem 6. An IFS on $\mathbb{R}$ does not satisfy OSC if and only if $\bar{J}=\mathbb{R}$.

Proof. We need only show that no OSC implies that $\bar{J}=\mathbb{R}$. Let $f_{i}(x)=r_{i} x+d_{i}$, $1 \leq i \leq m$. Without loss of generality, we may assume that $d_{1}=0$. It follows that $0 \in A$. Define $D=\{h(0) \mid h \in \mathcal{N}\}$.

First $D \subseteq \bar{J}$, for $h(0) \in h(A) \subset \bar{J}$ by Proposition 3. In the following, we will show that $\bar{D}=\mathbb{R}$ and hence $\bar{J}=\mathbb{R}$.

We can assume that $r_{1}>0$. Otherwise we consider the IFS formed by the $f_{i j}$ with $i, j \in S$ for which $J$ is not larger and $f_{11}(x)=r_{1}^{2} x$.

Take any neighbor map $h=f_{\mathbf{i}}^{-1} f_{\mathbf{j}}(x)$, where $f_{\mathbf{i}}^{-1}(x)=a_{1} x+b_{1}$ and $f_{\mathbf{j}}(x)=$ $a_{2} x+b_{2}$. Then $h(x)=a_{1} a_{2} x+a_{1} b_{2}+b_{1}$. Pick any $\delta>0$; we will show that there exists a neighbor map $h^{*} \in \mathcal{N}$ such that

$$
\delta / 2 \leq h^{*}(0)-h(0) \leq\left(1 / r_{1}+1 / 2\right) \delta .
$$

Let $g(x)=a x+b$ be a neighbor map in $\mathcal{N}$. Denote $g^{-1}(x)=x / a-b / a=a^{\prime} x+b^{\prime}$. Since there is no OSC, we can choose $g$ arbitrarily near to the identity map. Without loss of generality, we may assume that $a_{1} b>0$; otherwise we exchange $g$ and $g^{-1}$. We choose $g$ so near to the identity map that

$$
\left|(a-1) a_{1} b_{2}\right|<\delta / 2,\left|a_{1} b\right|<\delta \text { and }\left|\left(a^{\prime}-1\right) a_{1} b_{2}\right|<\delta / 2,\left|a_{1} b^{\prime}\right|<\delta .
$$

Let $g_{1}(x)=\left(f_{1}^{-1}\right)^{k} \cdot g \cdot f_{1}^{k}(x)$; then $g_{1}(x) \in \mathcal{N}$ and $g_{1}(x)=a x+r_{1}^{-k} b$. Choose the integer $k$ such that

$$
\delta \leq r_{1}^{-k} a_{1} b<\delta / r_{1}
$$

Let $h^{*}(x)=f_{\mathbf{i}}^{-1} \cdot g_{1} \cdot f_{\mathbf{j}}(x)$. Then

$$
\delta / 2<h^{*}(0)-h(0)=(a-1) a_{1} b_{2}+r_{1}^{-k} a_{1} b<\left(1 / r_{1}+1 / 2\right) \delta .
$$

Likewise let $g_{2}(x)=\left(f_{1}^{-1}\right)^{k} \cdot g^{-1} \cdot f_{1}^{k}(x)$, where $k$ is chosen such that $\delta \leq-r_{1}^{-k} a_{1} b^{\prime}<$ $\delta / r_{1}$. We get a map $h_{*}(x)=f_{\mathbf{i}}^{-1} \cdot g_{2} \cdot f_{\mathbf{j}}(x) \in \mathcal{N}$ such that

$$
\delta / 2 \leq h(0)-h_{*}(0) \leq\left(1 / r_{1}+1 / 2\right) \delta .
$$

Hence for any $\delta>0$ and any $x \in D$, there are two points $y_{1}, y_{2} \in D$ such that

$$
\delta / 2 \leq y_{1}-x \leq\left(1 / r_{1}+1 / 2\right) \delta, \quad \delta / 2 \leq x-y_{2} \leq\left(1 / r_{1}+1 / 2\right) \delta .
$$

Therefore $\bar{D}=\mathbb{R}$.

\section{REFERENCES}

[1] C. Bandt and S. Graf, Self-similar sets VII. A characterization of self-similar fractals with positive Hausdorff measure. Proc. Amer. Math. Soc. 114 (1992), 995-1001. MR 1100644 (93d:28014)

[2] K.J. Falconer, Fractal Geometry, Wiley, 1990. MR1102677(92j:28008)

[3] J.E. Hutchinson, Fractals and self-similarity, Indiana Univ. Math. J. 30 (1981), 713-747. MR0625600 (82h:49026) 
[4] M. Moran, Dynamical boundary of a self-similar set. Fund. Math. 160 (1999), 1-14. MR1694399 (2000f:28009)

[5] P.A.P. Moran, Additive functions of intervals and Hausdorff measure. Proc. Cambridge Philos. Soc. 42 (1946), 15-23. MR.0014397 (7:278f)

[6] A. Schief, Separation properties for self-similar sets. Proc. Amer. Math. Soc. 122 (1994), 111-115. MR.1191872 (94k:28012)

Institute for Mathematics, Arndt University, 17487 Greifswald, Germany

E-mail address: bandt@uni-greifswald.de

Institute for Mathematics, Arndt University, 17487 Greifswald, Germany

E-mail address: nvh0@yahoo.com

Department of Mathematics, Tsinghua University, P.O. Box 100084, Beijing, People's Republic of China

E-mail address: HRao@math.tsinghua.edu.cn 